\title{
Paramedic patient advocacy: a review and discussion
}

Alan M. Batt

Fanshawe College, London, Ontario, alan@batt.me

Gerard Ward

Royal College of Surgeons of Ireland, Dublin, Ireland

Joseph J. Acker

British Columbia Ambulance Service, BC, Canada

Follow this and additional works at: https://nsuworks.nova.edu/ijahsp

Part of the Interprofessional Education Commons

\section{Recommended Citation}

Batt AM, Ward G, Acker JJ. Paramedic patient advocacy: a review and discussion. The Internet Journal of Allied Health Sciences and Practice. 2017 Jan 01;15(4), Article 8.

This Review Article is brought to you for free and open access by the College of Health Care Sciences at NSUWorks. It has been accepted for inclusion in Internet Journal of Allied Health Sciences and Practice by an authorized editor of NSUWorks. For more information, please contact nsuworks@nova.edu. 


\title{
Paramedic patient advocacy: a review and discussion
}

\begin{abstract}
Introduction: Patient advocacy is an often encountered term, but one for which no consistent definition exists. Advocacy is a new and developing role of the paramedic that is closely linked to the developing professionalism of paramedicine, along with the expanding role of the paramedic as a healthcare professional in the community. This role however requires exploration and clarification. Methods: A literature search was performed of multiple databases, including MEDLINE, EMBASE, CINAHL and prominent paramedic journals. Results: No published literature investigating the theory or practice of paramedic patient advocacy, the potential conflicts and benefits of this role, or the perceived and actual barriers to advocacy paramedics face was discovered. Conclusion: The following literature review and discussion investigate the concept of paramedic patient advocacy, the contexts in which advocacy may take place, potential barriers, benefits and conflicts. Proposals are also included for areas requiring further research.
\end{abstract}

\section{Author Bio(s)}

Alan Batt MSc CCP is a critical care paramedic, educator and researcher based in Ontario, Canada.

Gerard Ward BSc CCP is a critical care paramedic and a medical student at the Royal College of Surgeons in Ireland, in Dublin, Ireland.

Joseph Acker MA EMT-P(cc) is a critical care paramedic, Director of Patient Care Delivery with British Columbia Ambulance Service, Canada and an Adjunct Senior Lecturer in Paramedicine at Charles Sturt University in NSW, Australia. 


\title{
TISAHSP \\ The Internet Joưnal of Allied Health Sciences and Practice \\ Dedicated to allied health professional practice and education
}

Vol. 15 No. 4 ISSN 1540-580X

\section{Paramedic Patient Advocacy: A Review and Discussion}

\author{
Alan M. Batt, MSc CCP 1 \\ Gerard Ward, BSc CCP2 \\ Joseph J. Acker, MA EMT-P(cc) ${ }^{3}$
}

1. Fanshawe College

2. Royal College of Surgeons of Ireland

3. British Columbia Ambulance Service

Ireland/Canada

\begin{abstract}
Introduction: Patient advocacy is an often encountered term, but one for which no consistent definition exists. Advocacy is a new and developing role of the paramedic that is closely linked to the developing professionalism of paramedicine, along with the expanding role of the paramedic as a healthcare professional in the community. This role however requires exploration and clarification. Methods: A literature search was performed of multiple databases, including MEDLINE, EMBASE, CINAHL and prominent paramedic journals. Results: No published literature investigating the theory or practice of paramedic patient advocacy, the potential conflicts and benefits of this role, or the perceived and actual barriers to advocacy that paramedics face was discovered. Conclusion: The following literature review and discussion investigate the concept of paramedic patient advocacy, the contexts in which advocacy may take place, potential barriers, benefits and conflicts. Proposals are also included for areas requiring further research.
\end{abstract}

\section{Introduction}

Patient advocacy is a term that is used frequently in healthcare settings, but it is one for which a consistent definition does not seem to exist. The documented definitions however centre on common themes of furthering the best interests, safety and wishes of the patient. A 2005 review identified the complexity of defining advocacy, ${ }^{1}$ and seventeen varying definitions were identified within the nursing literature alone. The concept of patient advocacy has been discussed in the nursing literature since the 1970s when the International Council of Nurses included it in their professional codes of conduct and ethics. It has been further refined, most recently in $2012 .{ }^{2}$ The nurse's role as a patient advocate is one that is underpinned by nursing ethics, another well researched and documented area of nursing professionalism.

The role of ambulance staff and especially that of paramedics has evolved substantially over the past 20 years, including expanded scopes of practice, the beginnings of paramedic-led clinical governance and increased clinical autonomy. In some health care systems being a patient advocate from a paramedic's perspective means transporting the patient to the hospital safely, and if possible beginning treatment in the prehospital setting. However in recent years in some services, advocating for one's patient can mean not transporting the patient to a hospital at all. Paramedics in many jurisdictions now have the ability to generate and implement treatment plans for patients with the option to transport, refer or discharge at scene, as deemed appropriate. With this evolution and role expansion has come greater responsibility, including an expanded scope of practice, and an obligation to patients to provide appropriate treatment, transport or discharge advice in the out-of-hospital setting. This has in turn required paramedics to advocate for their patients in both their decision making (for example, treat and discharge) and treatment options (for example, administration of medications).

Patient advocacy is a new, developing role of the paramedic that is closely linked to the developing professionalism of paramedicine. Paramedicine, though not formally defined in the existing literature, is the unique domain of education, practice and self-determination of paramedics, which includes traditional emergency response, and evolving non-emergent roles such 
as community paramedicine. This evolution can be evidenced through the increasing recognition of paramedics as regulated health professionals in countries such as the United Kingdom, Republic of Ireland, South Africa, Australia (forthcoming) and various provinces within Canada, and consequently, the inclusion of advocacy language in the national codes of ethical conduct of these jurisdictions. .-5 $^{3-5}$ The concept that patients require healthcare professionals to advocate for them does not seem to be in dispute; however, whether paramedics are best placed to become patient advocates is unknown.

Other healthcare professions, nursing in particular, have researched and published widely on the concept of advocacy in the context of their given profession. ${ }^{1}$ This is an important concept, as the practice of advocacy varies from one profession to the next due to inherent boundaries including clinical setting, clinical urgency, professional role and patient expectations. With the concept of ethical care at the forefront of teaching it is now more relevant than ever for the term of 'patient advocacy', especially in the context of paramedicine, to be defined and discussed.

\section{Aim}

Current peer-reviewed literature was sought surrounding the concept of paramedic patient advocacy, the role of the paramedic as a patient advocate, and any theoretical or practical models or constructs of paramedic patient advocacy.

\section{Methods and Data}

The search for articles focusing on paramedic patient advocacy was limited to peer-reviewed research published in Englishlanguage journals. The database searches in CINAHL, EMBASE and MEDLINE were based on the keywords 'patient advocacy/advocacy/advocate; paramedic/EMT' using MeSH headings where appropriate. No date restrictions were applied to ensure all relevant literature was captured in the review.

The initial search yielded 440 papers, 23 in MEDLINE, 390 in EMBASE and 27 in CINAHL. These were further reviewed independently by both authors for relevance and duplication. First, the titles and abstracts of the papers identified were examined. Editorials, guidelines, case studies, consensus statements, letters and commentaries were excluded, as were articles that addressed professional or political advocacy. A number of articles addressed certain aspects of patient advocacy but were written as online advice columns, advising paramedics on the practical aspects of how to be a patient advocate without discussing what this meant as an aspect of paramedicine, and thus were also excluded. Full texts were read for any article deemed potentially relevant to the question.

The archives of the Journal of Paramedic Practice (JPP), International Journal of Paramedic Practice (IPP), Australasian Journal of Paramedicine (AJP) and the Irish Journal of Paramedicine (IJP) were also searched for the terms "advocacy/patient advocacy/advocate". A total of 36 results were found in the JPP, 66 results in the IPP, 21 in the AJP and 0 in the IJP. The titles and abstracts of the papers identified were examined, and exclusion criteria were applied as per above.

A standard Google web search and a Google Scholar search were also performed using the terms "paramedic patient advocacy" and "patient AND advocate AND paramedic". A number of informal online columns and professional publications were again identified, as were excerpts from several paramedic textbooks.

\section{Results}

No published literature in peer-reviewed publications was discovered investigating the theory or practice of paramedic patient advocacy, defining patient advocacy from the perspective of paramedicine, or discussing theoretical or practical models of paramedic patient advocacy. The absence of peer-reviewed literature discussing patient advocacy in paramedicine from the sources reviewed suggests a critical absence of this topic from the forums of scholarly communication employed by paramedics, medical supervisors, and governing and oversight bodies. Several professional publications (not peer reviewed) were identified in publications such as The Journal of Emergency Medical Services and Canadian Paramedicine.

\section{Discussion}

While no peer-reviewed papers were found in the literature search which provided detail on paramedic patient advocacy, many professional papers addressed or proposed themes that can be discussed further to highlight areas for potential future research. The search did highlight several articles and online blogs discussing how paramedics could advocate for their patients, with reliable and practical advice outlined. This lack of peer-reviewed literature, but an abundance of professional literature is a symptom of the infancy of research within paramedicine, although this is slowly changing. None of these professional articles or blog posts however discussed the concept of advocacy from the perspective of paramedicine, and rather provided practical advice to paramedics on advocacy activities.

(C) The Internet Journal of Allied Health Sciences and Practice, 2017 
To guide and frame our discussion, we refer to the extensive literature on advocacy in other healthcare professions to guide the development of the paramedic as a patient advocate. There exist a number of articles and reviews of the literature surrounding theory, practice and models of nursing advocacy. ${ }^{1,6}$

Advocacy in nursing can be comprised and broken down into three distinct themes; activities related to educating the patient, assisting and supporting decision-making (proactive advocacy); activities related to safeguarding the patient's privacy, confidentiality, safety, and rights (reactive advocacy); and the concept of advocacy as a principle of nursing practice. ${ }^{6}$ Given some conceptual overlap between the patient-caregiver relationship for the nursing and paramedic professions, exploration of the validity of these dimensions of advocacy in paramedicine is warranted.

The role of the paramedic as a patient advocate is not discussed in the literature, adding a further layer of complexity to defining 'paramedic patient advocacy'. With the introduction of community care and paramedic practitioner programs the traditional role of a paramedic has evolved and modern paramedics find themselves in a unique position in that treat and refer options can now be implemented for non-critical, chronic patients and for patients whose best interest is not being admitted to an emergency department.

Some of the initial findings in the 'High Quality Care For All' report, an extensive review of the NHS care system as a whole published in 2008 , found that patients should receive "health care services closer to home" and furthermore a "personalized care plan" should be implemented for each individual patient. ${ }^{7}$ This report highlights patient advocacy as a key pillar related to patients and highlights that health care practitioners at all levels should always aim for this when caring for patients. For paramedics, this could be interpreted as a challenge to the traditional view of an 'ambulance service' and its role as a transfer tool for the community.

Community and paramedic practitioners are now in a position to be true patient advocates and make decisions based on the patient's needs rather than standard operating procedures for ambulance services, i.e. to transport the patient and treat them on the way to a hospital. In some cases the patient can now be referred to an out-patient or step-down unit which can have positive cascading effects by decreasing waiting times within hospitals and in Emergency Departments.

\section{Advocacy Contexts}

The very nature of paramedicine puts paramedics in a unique position to advocate for the patient. With the privilege of entering patient's homes comes exposure to first-hand knowledge of the patient's social conditions, presentation at the scene, environmental conditions and other factors. What truly distinguishes paramedics from other healthcare professionals are the settings in which they practice ${ }^{8}$, and while commonly associated with emergency medical care, the practice of paramedicine has expanded significantly beyond the constraints of the ambulance. Community paramedicine initiatives that have been established in Canada, the USA, UK and other regions have placed paramedics in non-traditional settings, providing long-term and continuing care to vulnerable populations. ${ }^{9}$ Paramedics also provide care in remote and rural health settings ${ }^{10}$, industrial and commercial settings ${ }^{8,11}$, on offshore platforms and vessels ${ }^{8}$, during expeditions in extreme climates ${ }^{12}$ and many other varied settings.

Advocacy is usually employed by someone powerful on behalf of someone who has no power, in situations of powerlessness, difficult circumstances, or vulnerability. ${ }^{13}$ Paramedics provide care for patients with acute medical and trauma presentations, such as domestic violence, child mistreatment and elder abuse, the homeless, patients in remote settings far from definitive care, mental health issues, substance abuse, ill or injured incarcerated persons, terminally ill patients, and chronic disease presentations. As can be seen from this list, paramedics regularly encounter individuals experiencing lack of power, difficult circumstances and who are vulnerable, and thus may potentially be in a position as a profession to advocate for a large portion of the population.

Previous studies have already proven that paramedics can serve an important role in identifying at-risk elderly patients and can begin the process of referral to social services. ${ }^{14,15}$ Homeless people in a study conducted in Toronto were far more likely to seek the help of, and trusted paramedics substantially more than police..$^{16} \mathrm{~A}$ study in Denver, Colorado found that homeless patients presenting to an Emergency Department were far more likely to utilise ambulance services, even though $90 \%$ of these transports were non-urgent in nature. ${ }^{17}$ In the context of dealing with mental health emergencies, including deliberate self-harm, paramedics have also been shown to play an important role. ${ }^{18}$ 
Paramedics in several jurisdictions have indicated that interacting with, and caring for terminally ill patients is a common occurrence in clinical practice. Guru et al. discovered that paramedics frequently encountered ethical dilemmas when caring for terminally ill patients in cardiac arrest, and even breached protocols in order to provide care for their patients in these cases. ${ }^{19}$ Several studies indicate a need for further education and organisational support for paramedics in providing adequate care for this patient population ${ }^{20-22}$. Thus it can be seen that the social and clinical contexts in which patient advocacy from the perspective of paramedicine needs to be studied are wide-ranging, and may differ significantly across territories.

\section{Foundations}

The foundations for proposing the concept of paramedic patient advocacy exist in many countries. Paramedics are registered health professionals in many territories as outlined earlier, or are alternatively represented by professional associations. This professional regulation or representation generally requires a paramedic to agree to abide by a code of conduct or ethics which either explicitly state the role of being a patient advocate, or address many activities of the patient advocate role. ${ }^{3-5}$ These codes of conduct and ethics are based on the core principles of medical ethics, namely respect for autonomy, beneficence, nonmaleficence and justice ${ }^{23}$; essential underpinning aspects of patient advocate behaviour and activities.

Educational curricula for paramedic education generally deliver information on key patient advocacy activities that the paramedic must have knowledge of and demonstrate in practice. However, the theory and practice of patient advocacy as a fundamental role of the paramedic is not well represented across paramedic curricula. The term 'patient advocate' seems to be used freely within many paramedic educational curricula and standards without there being an adequate definition that can be fully applied to paramedicine. Instead, this term is used as a broad label for activities the paramedic can perform to advocate for their patient, for example maintaining privacy and confidentiality and having the patient's best interests in mind.

As a result of this, there exists an additional requirement for further research into the initial and continuing education requirements for paramedics with regards to ethical behaviour, ethical dilemmas, patient advocacy and patient education. This is not unique to paramedicine. Several other professions, including medicine, have identified a need for clearer objectives in teaching advocacy. ${ }^{24}$

\section{Potential Barriers to Advocacy}

This review identified no readily-available published literature which discusses the actual and perceived barriers to paramedic patient advocacy, and thus the theoretical barriers proposed here by the authors cannot be said to be representative of all paramedics in all jurisdictions. A number of individual themes that potentially hinder the role of the paramedic as a patient advocate were however highlighted during the review of the patient advocacy literature, albeit these articles did not discuss paramedic patient advocacy itself.

Paramedics potentially face many similar barriers as nurses to being a true patient advocate - lack of support from management and colleagues (powerlessness), fear of litigation (code of ethics, law), professional boundaries with other healthcare professionals, and at times, lack of motivation or ability, due to resources, time or education. ${ }^{25,26}$ Paramedics can be clinically restricted as they often follow defined clinical practice guidelines, which in some jurisdictions cannot be deviated from unless approved by a physician. Contact with physicians is sometimes not possible and so the patient may be transferred to a health care facility for further observation or consultation unnecessarily.

The concept of "knowing the patient" 27,28 is an area where paramedics face a significant barrier to realising their role as patient advocates, as insufficient time with patients can hamper communication. As a paramedic, time spent caring for a patient is often fleeting, and happens in an unpredictable and sometimes chaotic environment. The authors hypothesise that this unpredictability can make it more difficult to establish a positive therapeutic relationship. However, Ahl and Nystrom found that having a one-toone relationship with their prehospital clinician enhanced patients' feelings of security, which seems to increase acceptance and participation in the therapeutic relationship on the patient's behalf. ${ }^{29}$

There is presently a worldwide shortage of paramedics and ambulance staff. NHS Workforce figures show that in 2015 the NHS was operating at $63 \%$ of the required minimum ambulance staffing levels and there were $10.4 \%$ staff vacancies for minimum paramedic staffing levels. ${ }^{30}$ These figures show a growing trend of a huge strain on paramedics and other prehospital care staff and may seriously decrease the face to face time that paramedics can spend with each patient due to limited resources. This in turn can impact the paramedic's ability to be a true patient advocate and in most cases, can have the opposite effect and mean the paramedic will be forced to revert back to acting as a transport service due to time restraints and call volumes.

(c) The Internet Journal of Allied Health Sciences and Practice, 2017 
Empathy is widely recognised as an integral component in establishing positive therapeutic relationships. Recent findings have highlighted that paramedic students displayed lower levels of empathy than nursing or midwifery students. ${ }^{31-33}$ This highlights an area for potential improvement, as ensuring the development of paramedics with appropriate levels of empathy may improve communication with patients, and thus the establishment of therapeutic relationships. Development of empathy may be possible through the integration of empathy teaching and learning, and appropriate development and assessment of nontechnical skills in paramedic students during clinical simulation scenarios.

The perspectives of paramedics and the actual and conceived barriers that exist to paramedic patient advocacy warrant research to inform the role of the paramedic as a patient advocate.

\section{Potential Benefits \& Conflict}

The potential benefits for paramedics in pursuing a stronger professional identity as patient advocates could come in the form of increased collaboration, proficiency and professional autonomy. The exact benefits are unknown, but similar benefits have been described by nursing and other professions in their role as patient advocates. ${ }^{1,34}$

Pursuing a stronger role as a patient advocate could also potentially cause some conflict for paramedics. As identified by O'Connor and Kelly in 2005, the patient advocacy role resulted in nurses becoming involved in conflict and confrontation with others, and patient advocacy could be detrimental to nurses both professionally and personally. ${ }^{35}$ Other potential negative consequences identified on a review of the nursing advocacy literature included loss of status, job or professional role for the advocate, and direct or indirect conflict with the organisation. ${ }^{1}$ Given the high stress levels already identified among paramedics ${ }^{36}$, the addition of this potential conflict may be detrimental to the individual paramedic.

Vaartio and Leino-Kilpi have identified a lack of research into nursing advocacy from the perspective of the patients. ${ }^{1}$ Does advocacy mean supporting any decision the patient makes, or representing the patient by asserting well-intentioned paternalistic claims on their behalf? Paramedics should not pursue a role as a patient advocate for purely professional purposes; this is the antithesis of the role of an advocate. As stated earlier, varied definitions of advocacy exist, and an accepted definition applicable to paramedicine needs to be identified before additional meaningful research can be conducted. Based on our reading of the advocacy literature in other healthcare professions, we would propose the definition "professional action by a paramedic in order to support or defend a patients' rights and wellbeing."

A study by Ahl and Nystrom in 2012 explored the perceptions of patients after interacting with emergency services after an emergency call was placed. ${ }^{29}$ Participants stated a sense of security knowing that an ambulance was on its way and stated they felt cared for by providers. Some participants claimed they had never felt so respected, believed in and listened to as they did when interacting with prehospital care providers, ${ }^{29}$ and similar themes were found in a previous study by Elmqvist et al. in $2008 .{ }^{37}$ It should however be noted that both of these studies were conducted in Sweden, where nurses are the primary prehospital care providers. The authors propose that similar studies should be considered in territories where paramedics are the primary prehospital clinician.

The consequences of paramedic patient advocacy need to be quantified to determine if this expanded role is one that is warranted and justified by the impact it has on patients, and their perceptions of advocacy activities by paramedics. It is unclear from most nursing advocacy literature whether the patients experienced themselves as oppressed, or whether they requested or recognised advocacy activities by nurses. The authors propose that paramedic patient advocacy needs to encompass a variety of underlying principles, education, relationships and a balance of the patient needs (clinical, social, psychological etc.) versus the patient rights (autonomy, right to bodily integrity, refusal of care etc.).

\section{Limitations}

This review discovered no published peer-reviewed literature that investigated the theory or practice of paramedic patient advocacy, defined patient advocacy from the perspective of paramedicine, or discussed theoretical or practical models of paramedic patient advocacy. As such the themes, benefits, conflicts and issues arising cannot be stated as accurate or totally representative of the actual issues surrounding the role of the paramedic as a patient advocate. However, themes that were prevalent in grey literature, and the literature relating to other professions, have been referenced to allude to potential areas for discussion and further research. These items are put forward by the authors based on their reading of the wider advocacy literature.

(c) The Internet Journal of Allied Health Sciences and Practice, 2017 


\section{Conclusion}

The role of the paramedic as a patient advocate does not appear to have been researched to date. The term patient advocate with reference to prehospital care staff, and more specifically to paramedics, needs to be further explored and defined. Presently it is used as a general term for 'better patient care'. Paramedics need to be enabled and supported from professional, educational, clinical, legal and organisational perspectives before their true potential role as a patient advocate can be realised. In order to advance the role of the paramedic as a patient advocate, the barriers, benefits, and conflicts associated with this role require further research before being fully pursued by the paramedic profession.

\section{References}

1. Vaartio H, Leino-Kilpi H. Nursing advocacy-a review of the empirical research 1990-2003. Int J Nurs Stud [Internet]. 2005;42(6):705-14. Available from: http://www.ncbi.nlm.nih.gov/pubmed/15982468 [PMID: 15982468]

2. International Council of Nurses. The ICN Code of Ethics for Nurses [Internet]. 2012. Available from: http://www.icn.ch/images/stories/documents/about/icncode_english.pdf. Accessed June 20, 2017.

3. Health and Care Professions Council. Standards of Conduct, Performance and Ethics [Internet]. London; 2014. Available from: www.hpc-uk.org. Accessed June 20, 2017.

4. Pre-Hospital Emergency Care Council. Code of Conduct and Ethics. Kildare; 2008.

5. Health Professions Council of South Africa. General Ethical Guidelines for the Health Care Professions. 2007.

6. Mallik M. Advocacy in nursing--a review of the literature. J Adv Nurs. 1997;25(1):130-8. [PMID: 9004021]

7. Darzi A. High Quality Care For All [Internet]. London; 2008. Available from: http://www.formativexperience.com/upload/High Quality Care for All.pdf. Accessed June 20, 2017.

8. Acker JJ, Johnston TJ, Lazarsfeld-Jensen A. Industrial paramedics, out on site but not out of mind. Rural Remote Health. 2014;14(4):1-17. [PMID: 25480308]

9. O'Meara P, Ruest M, Stirling C. Community paramedicine: higher education as an enabling factor. Australas J Paramed [Internet]. 2014;11(2). Available from: http://ro.ecu.edu.au/jephc/vol11/iss2/5/. Accessed June 20, 2017.

10. Stirling CM, O'Meara P, Pedler D, Tourle V, Walker J. Engaging rural communities in health care through a paramedic expanded scope of practice. Rural Remote Health. 2007;7(4):839. [PMID: 18062741]

11. Seel D, Turner M. Industrial paramedic: an emerging speciality? J Paramed Pract. 2016;8(7):350-5.

12. Allen SH, Moore J, Grocott MPW. Expedition medicine in the tropics: through heat and sleet. Trans $R$ Soc Trop Med Hyg. 2009;103(11):1081-4. [PMID: 19740504]

13. Teasdale K. Advocacy in Health Care. Oxford: Wiley; 1998.

14. Kue R, Ramstrom E, Weisberg S, Restuccia M. Evaluation of an Emergency Medical Services-Based Social Services Referral Program for Elderly Patients. Prehospital Emerg Care. 2009;13(3):273-9.

15. Gerson LW, Schelble DT, Wilson JE. Using paramedics to identify at-risk elderly. Ann Emerg Med. 1992;21(6):688-91. [PMID: 1590608]

16. Zakrison TL, Hamel PA, Hwang SW. Homeless people's trust and interactions with police and paramedics. J Urban Heal. 2004;81(4):596-605. [PMID: 15466841]

17. Pearson D, Bruggman AR, Haukoos JS. Out-of-Hospital and Emergency Department Utilization by Adult Homeless Patients. Ann Emerg Med. 2007;50(6):646-52. [PMID: 17950488]

18. O'Sullivan B. Paramedic attitudes to deliberate self-harming behaviour in Ireland. J Paramed Pract. 2010;6(3):116-8.

19. Guru V, Verbeek PR, Morrison LJ. Response of paramedics to terminally ill patients with cardiac arrest: an ethical dilemma. CMAJ. 1999;161(10):1251-4. [PMID: 10584085]

20. Kirk A, Crompton $P$, Jack B, Knighting K, Kirt J. A scoping exercise to explore the current perceptions and attitudes of UK Paramedics towards their role in the management of end of life care patients, in the pre-hospital setting. In: The 10th Palliative Care Congress. 2014. p. A7-8.

21. Waldrop DP, Clemency B, Maguin E, Lindstrom H. Preparation for frontline end-of-life care: exploring the perspectives of paramedics and emergency medical technicians. J Palliat Med. 2014;17(3):338-41. [PMID: 24517266]

22. Wiese C, Taghavi M, Meyer N, Lassen C, Graf B. Paramedics' "end-of-life" decision making in palliative emergencies. J Paramed Pract. 2012;4(7):143-68.

23. Gillon R. Medical ethics: four principles plus attention to scope. BMJ. 1994;309(6948):184-8. [PMID: 8044100]

24. Verma S, Flynn L, Seguin R. Faculty's and residents' perceptions of teaching and evaluating the role of health advocate: a study at one Canadian university. Acad Med. 2005;80(1):103-8. [PMID: 15618104]

25. Negarandeh R, Oskouie F, Ahmadi F, Nikravesh M, Hallberg IR. Patient advocacy: barriers and facilitators. BMC Nurs [Internet]. 2006;5(1):3. Available from: http://www.biomedcentral.com/1472-6955/5/3 [PMID:16509969]

26. Moloney B, Batt A. The paramedic as a patient advocate. Can Paramed. 2015;38(6):16-9.

27. Whittemore R. Consequences of not "knowing the patient". Clin Nurse Spec. 2000;14(2):75-81. PMID:11188452

(C) The Internet Journal of Allied Health Sciences and Practice, 2017 
28. Tanner CA, Benner P, Chesla C, Gordon DR. The Phenomenology of Knowing the Patient. Image J Nurs Scholarsh. 1993;25(4):273-80.

29. Ahl C, Nyström M. To handle the unexpected - The meaning of caring in pre-hospital emergency care. Int Emerg Nurs. 2012;20(1):33-41. [PMID: 22243716]

30. National Health Executive. Paramedic Workforce Shortfall. 2015.

31. Nunes $P$, Williams $S$, Sa B, Stevenson K. A study of empathy decline in students from five health disciplines during their first year of training. Int $J$ Med Educ. 2011;2:12-7.

32. Williams B, Boyle M, Tozer-Jones J, Devenish S, Hartley P, et al. Undergraduate paramedic students' empathy levels: A two-year longitudinal study. J Nurs Educ Pract. 2014;5(1).

33. Williams B, Brown T, Boyle M, McKenna L, Palermo C, Etherington J. Levels of empathy in undergraduate emergency health, nursing, and midwifery students: a longitudinal study. Adv Med Educ Pract. 2014;5:299-306. [PMID: 25246815]

34. Bernal EW. The Nurse as Patient Advocate. Hastings Cent Rep. 1992;22(4):18-23.

35. O'Connor T, Kelly B. Bridging the gap: a study of general nurses' perceptions of patient advocacy in Ireland. Nurs Ethics. 2005;12(5):453-67. [PMID: 16178342]

36. Mildenhall J. Occupational stress, paramedic informal coping strategies: a review of the literature. $J$ Paramed Pract. 2012;4(6):318.

37. Elmqvist C, Fridlund B, Ekebergh M. More than medical treatment: The patient's first encounter with prehospital emergency care. Int Emerg Nurs. 2008;16(3):185-92. [PMID: 18627804] 